\title{
UNIQUENESS OF THE OPEN CONE NEIGHBORHOOD
}

\section{KYUNG WHAN KWUN ${ }^{1}$}

1. Introduction. The space $A \times[0, \infty)$ with $A \times 0$ identified to a point $v$ is called the open cone $O C(A)$ over $A$ and the point $v$ is called the vertex of the cone.

Let $X$ be a space. A point $x \in X$ is said to have an open cone neighborhood $U$ if there is a homeomorphism $f$ of some $O C(A)$ onto the open set $U$ of $X$ with $f(v)=x$. Our first theorem is the following.

THEOREM 1. Let $U$ and $V$ be any two open cone neighborhoods of a point $x$ in a locally compact Hausdorff space $X$. Then there is a homeomorphism of $V$ onto $U$ which leaves a neighborhood of $x$ pointwise fixed.

As immediate corollaries, we obtain a result of Mazur and Rosen that the open star of a vertex of a triangulated manifold is an open cell and also a result of Kwun and Raymond that the open star of a vertex on the boundary of a triangulated manifold with boundary is a cell minus a boundary point.

Theorem 1 was discovered when we tried to prove the following:

Theorem 2. Let $M$ be a compact manifold with boundary. If $M^{\prime}$ is a compact manifold with boundary such that Int $M=\operatorname{Int} M^{\prime}$ then $\mathrm{Bd} M$ $\times E^{1}=\mathrm{Bd} M^{\prime} \times E^{1}$. Conversely, if $B$ is a compact manifold such that Bd $M \times E^{1}=B \times E^{1}$ then there exists a compact manifold $M^{\prime}$ with boundary such that Int $M^{\prime}=$ Int $M$ and $\mathrm{Bd} M^{\prime}=B$.

Unfortunately, we do not know if $\mathrm{Bd} M \times E^{1}=\mathrm{Bd} M^{\prime} \times E^{1}$ does not imply $\mathrm{Bd} M=\mathrm{Bd} M^{\prime}$.

Finally the method of the proof of Theorem 1 may be used to prove more general theorems. For example,

Theorem 3. With $x$ and $X$ as in Theorem 1 , if $U^{1} \subset U^{2} \subset \cdots$ is a sequence of open cone neighborhoods of $x$ then $U=U U^{i}$ is also an open cone neighborhood of $x$ homeomorphic to each $U^{i}$.

This generalizes [1].

2. Proof of Theorem 1. Let $f: O C(A) \rightarrow X$ and $g: O C(B) \rightarrow X$, be homeomorphisms defining $U$ and $V$ respectively as open cone neighborhoods of $x$. Local compactness of $X$ implies compactness of $A$ and 1962.

Presented to the Society, November 17, 1962; received by the editors October 16,

1 Supported in part by NSF GP-626. 
$B$. Hence we may assume that $X=U=O C(A)$ and $f$ is the identity. We express each point of $O C(A)$ by $(a, t), a \in A, t \geqq 0$ with the understanding that $A \times 0$ is identified to $x$. The set $A \times t$ is abbreviated to $A_{\imath}$. We do similarly for $O C(B)$ and denote by $U_{t}$ and $V_{t}$ respectively the compact sets $U_{t^{\prime} \leqq t} A_{t^{\prime}}$ and $U_{t^{\prime} \leqq t} g\left(B_{t^{\prime}}\right)$. Observe that if $t>0$, there exist $t^{\prime}, t^{\prime \prime}>0$ such that $V_{t^{\prime}} \subset U_{t}$ and $U_{t^{\prime \prime}} \subset V_{t}$.

1. We find five positive numbers $p<q<r, s<t$ such that $g\left(B_{s}\right)$ separates $A_{p}$ from $A_{q}$ and $g\left(B_{t}\right)$ separates $A_{q}$ from $A_{r}$. This is done by a repeated use of the above observation.

2. There exists a homeomorphism $h_{0}$ of $A \times[p, q]$ onto $A \times[q, r]$ such that

$$
\begin{gathered}
h_{0}(a, p)=(a, q), \\
h_{0}(a, q)=(a, r), \\
h_{0} g(b, s)=g(b, t) .
\end{gathered}
$$

In particular, there is a homeomorphism $F$ of $B \times[1,2]$ into $A \times[p, r]$ such that

$$
F(b, 1)=g(b, s) \text { and } F(b, 2)=h_{0} g(b, s) .
$$

This can be easily seen as follows. Let $U_{s}^{\prime}$ and $V_{s}^{\prime}$ denote the sets $\mathrm{U}_{s^{\prime} \geq s} A_{s^{\prime}}$ and $\mathrm{Cl}\left(U-V_{s}\right)$. We denote by $\epsilon$ a sufficiently small positive number. There exists a homeomorphism $k_{1}$ of $U$ onto itself such that $k_{1} \mid U_{q-\epsilon} \cup U_{r+\epsilon}^{\prime}=1$ and $k_{1}(a, q)=(a, r)$. There exists a homeomorphism $k_{2}$ of $U$ onto itself such that $k_{2} \mid V_{s-\epsilon} \cup V_{t+\epsilon}^{\prime}=1$ and $k_{2} g(b, s)=g(b, t)$. There exists a homeomorphism $k_{3}$ of $U$ onto itself such that $k_{3} \mid U_{\text {p-e }}$ $\cup U_{q+\epsilon}^{\prime}=1$ and $k_{3}(a, p)=(a, q)$. Let $h_{0}$ be the appropriate restriction of $k_{3} k_{2} k_{1}$.

3. Choose positive numbers $u_{i}, w_{j}$ such that

$$
\begin{aligned}
& q=u_{0}<r=u_{1}<u_{2}<\cdots, \\
& s=w_{0}<w_{1}<w_{2}<\cdots
\end{aligned}
$$

and

$$
\lim u_{i}=\lim w_{i}=+\infty .
$$

4. Let $H_{0}$ be the identity map of $V_{w_{0}}$. This can be extended to a homeomorphism $H_{1}$ of $V_{w_{1}}$ onto $U_{u_{0}} \cup_{g}(B \times[s, t])$.

5. Find a homeomorphism $h_{1}$ of $A \times\left[p, u_{1}\right]$ onto $A \times\left[q, u_{2}\right]$ which is an extension of $h_{0}$ such that $h_{1}\left(a, u_{1}\right)=\left(a, u_{2}\right)$.

Let $H_{2}$ be an extension of $H_{1}$ which maps $V_{w_{2}}$ homeomorphically onto $U_{u_{1}} \cup h_{1} g(B \times[s, t])$. ( $H_{2}$ can be chosen so that $H_{2}\left(g\left(b, w_{2}\right)\right)$ $=h_{1} g(b, t)$, but this is not necessary.) 
6. Find a homeomorphism $h_{2}$ of $A \times\left[u_{0}, u_{2}\right]$ onto $A \times\left[u_{1}, u_{3}\right]$ which is an extension of $h_{1} \mid A \times\left[u_{0}, u_{1}\right]$ such that $h_{2}\left(a, u_{2}\right)=\left(a, u_{3}\right)$.

Let $H_{3}$ be an extension of $H_{2}$ which maps $V_{w_{3}}$ homeomorphically onto $U_{u_{2}} \cup h_{2} h_{1} g(B \times[s, t])$.

7. Similarly, find $H_{4}, H_{5}, \cdots$ which define a homeomorphism of $V$ onto $U$ which leaves $V_{u_{0}}$ pointwise fixed.

\section{Proof of Theorem 2.}

The first part. Let $M^{*}$ and $M^{\prime *}$ be obtained from $M$ and $M^{\prime}$ by shrinking $\mathrm{Bd} M$ and $\mathrm{Bd} M^{\prime}$ to points $p$ and $p^{\prime}$ respectively. By [2], $p$ and $p^{\prime}$ have open cone neighborhoods homeomorphic to $O C(\mathrm{Bd} M)$ and $O C\left(\mathrm{Bd} M^{\prime}\right)$. Since $M^{*}$ and $M^{\prime *}$ are one-point compactifications of homeomorphic spaces Int $M$ and Int $M^{\prime}$, there is a homeomorphism of $M^{*}$ onto $M^{\prime *}$ under which $p$ is mapped into $p^{\prime}$. By Theorem $1, O C(\mathrm{Bd} M)=O C\left(\mathrm{Bd} M^{\prime}\right)$ with the vertices corresponding to each other. After deleting the vertices, $\mathrm{Bd} M \times E^{1}=\mathrm{Bd} M^{\prime} \times E^{1}$.

The second part. It follows that $O C(\mathrm{Bd} M)=O C(B)$ with the vertices of the cones corresponding. Hence, an examination of a set like $V_{s} \cap U_{p}^{\prime}$, where $V_{s}$ and $U_{p}^{\prime}$ are the sets defined in the proof of Theorem 1 and $s, p$ are positive numbers as in 2 of the proof of Theorem 1 , reveals the existence of a compact manifold $Y$ with boundary such that $\mathrm{Bd} Y$ is the disjoint union of $B$ and $\mathrm{Bd} M$ and $Y-B=\mathrm{Bd} M$ $\times[0,1)$ with $y \in \mathrm{Bd} M$ corresponding to $y \times 0$ and $Y-\mathrm{Bd} M=B$ $\times[0,1)$ with the points of $B$ similarly corresponding.

We let $M^{\prime}=M \cup Y$ with $M \cap Y=\mathrm{Bd} M$. Then clearly, $M^{\prime}$ is a compact manifold with boundary $B$. That Int $M^{\prime}$ is homeomorphic to Int $M$ follows from [2].

4. Proof of Theorem 3. Let $f^{i}: O C\left(A^{i}\right) \rightarrow X$ be homeomorphisms defining $U^{i}$ as open cone neighborhoods of $x$. As in the proof of Theorem $1, A_{t}^{i}$ denotes the subset $\left\{\left(a^{i}, t\right) \mid a^{i} \in A^{i}\right\}$ of $O C\left(A^{i}\right)$ and $U_{t}^{i}$ $=\bigcup_{t^{\prime} \leqq t} f^{i}\left(A_{t^{\prime}}^{i}\right)$.

We can find, one by one, positive numbers $t_{i j}, i, j=1,2, \cdots$ such that

$$
\begin{aligned}
& \bigcup_{j} U_{t_{i j}}^{i}=U^{i} \\
& U_{t_{i j}}^{i} \subset U_{t_{i+1, j}}^{i+1}-f^{i+1}\left(A_{t_{i+1}, j}^{i+1}\right)
\end{aligned}
$$

for each $i$ and $j$, and

$$
U_{t_{i j}}^{i} \subset U_{t_{i, j+1}}^{i}-f^{i}\left(A_{t_{i, j+1}}^{i}\right) .
$$

In what follows, $U_{t_{i}}^{i}$, etc. will be denoted simply by $U(i, j)$, etc. 
Clearly for any sequence $j_{1}<j_{2}<\cdots$ of positive integers $j_{i}$, $U U\left(i, j_{i}\right)=U$.

We will repeatedly use the method of the proof of Theorem 1.

Choose $j_{1}$. Let $H_{1}$ be the identity map of $U\left(1, j_{1}\right)$. Choose $j_{2}>j_{1}$. As in 2 and 5 of the proof of Theorem 1 , we extend $H_{1}$ to a homeomorphism $H_{2}$ of $U\left(1, j_{2}\right)$ onto a compact set $F_{2}$ containing $U\left(2, j_{2}\right)$ and contained in $U^{2}$. The next step reveals the general step. Since $H_{2}\left(f^{1}\left(A\left(1, j_{2}\right)\right)\right)$ has a product neighborhood in $U^{2}$, we extend $H_{2}$ to a homeomorphism $H_{2}^{\prime}$ of $U^{1}$ into $U^{2}$. Consider the open cone neighborhood $V^{1}$ defined by $H_{2}^{\prime} f^{1}: O C\left(A^{1}\right) \rightarrow X$. We find an integer $j_{3}>j_{2}$ so that $U\left(3, j_{3}-1\right)$ contains $F_{2}$. We extend the homeomorphism $H_{2}: V\left(1, j_{2}\right) \rightarrow F_{2}$ to a homeomorphism $H_{3}^{\prime}$ of $V\left(1, j_{3}\right)$ onto a compact set $F_{3}$ containing $U\left(3, j_{3}\right)$ and contained in $U^{3}$. Using $H_{3}^{\prime}$, we define a homeomorphism $H_{3}$, an extension of $H_{2}$, of $U\left(1, j_{3}\right)$ onto $F_{3}$. In exactly the same manner, we find $H_{3}, H_{4}, \cdots$ and they together generate a homeomorphism, leaving $U\left(1, j_{1}\right)$ pointwise fixed, of $U^{\prime}$ onto $U_{i} F_{i}=U$

5. Remarks. As we mentioned earlier, we do not know of any compact nonhomeomorphic manifolds $B$ and $B^{\prime}$ such that $B \times E^{1}=B^{\prime} \times E^{1}$. Although the nonexistence, if proved, would strengthen Theorem 2, one might feel that such $B$ and $B^{\prime}$ exist. One possibility is that $L(7,1) \times S^{n} \neq L(7,2) \times S^{n}$ for some $n$. Since $L(7,1) \times E^{n}=L(7,2) \times E^{n}$ for $n>2$ by [3], $\operatorname{Int}\left(L(7,1) \times I^{n}\right)=\operatorname{Int}\left(L(7,2) \times I^{n}\right)$ for $n>2$. Hence, by Theorem $2, L(7,1) \times S^{n-1} \times E^{1}=L(7,2) \times S^{n-1} \times E^{1}$. But the remaining question is whether $L(7, i) \times S^{n-1}$ are homeomorphic.

Also in Theorem 3, we need not assume $U$ to be the monotone union. It suffices to assume that $U_{t}^{i}, i=1,2, \cdots, t>0$, form a cofinal family in the collection of the compact subsets of $U$. Hence the proof of Theorem 3 implies a result due to Stallings [4].

Finally, we wish to thank J. Andrews for a stimulating conversation we had with him which incidentally was the start of the present work.

\section{REFERENCES}

1. Morton Brown, The monotone union of open n-cells is an open n-cell, Proc. Amer. Math. Soc. 12 (1961), 812-814.

2. - Locally flat embeddings of topological manifolds, Ann. of Math. (2) 75 (1962), 331-341.

3. John Milnor, Two complexes which are homeomorphic but combinatorially distinct, Ann. of Math. (2) 74 (1961), 575-590.

4. John Stallings, Groups with infinite products, Bull. Amer. Math. Soc. 68 (1962), 388-389.

Florida State University 